\title{
A deterministic and stochastic model for the system dynamics of tumor-immune responses to chemotherapy
}

DOI:

10.1016/j.physa.2018.02.118

\section{Document Version}

Accepted author manuscript

Link to publication record in Manchester Research Explorer

\section{Citation for published version (APA):}

Liu, X., Li, Q., \& Pan, J. (2018). A deterministic and stochastic model for the system dynamics of tumor-immune responses to chemotherapy. Physica A: Statistical Mechanics and its Applications, 500, 162-176.

https://doi.org/10.1016/j.physa.2018.02.118

\section{Published in:}

Physica A: Statistical Mechanics and its Applications

\section{Citing this paper}

Please note that where the full-text provided on Manchester Research Explorer is the Author Accepted Manuscript or Proof version this may differ from the final Published version. If citing, it is advised that you check and use the publisher's definitive version.

\section{General rights}

Copyright and moral rights for the publications made accessible in the Research Explorer are retained by the authors and/or other copyright owners and it is a condition of accessing publications that users recognise and abide by the legal requirements associated with these rights.

\section{Takedown policy}

If you believe that this document breaches copyright please refer to the University of Manchester's Takedown Procedures [http://man.ac.uk/04Y6Bo] or contact uml.scholarlycommunications@manchester.ac.uk providing relevant details, so we can investigate your claim.

\section{OPEN ACCESS}




\title{
A deterministic and stochastic model for the system dynamics of tumor-immune responses to chemotherapy
}

\author{
Xiangdong $\mathrm{Liu}^{\mathrm{a}}$, Qingze $\mathrm{Li}^{\mathrm{b}}$, Jianxin $\mathrm{Pan}^{\mathrm{b}, *}$

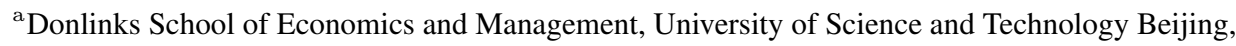 \\ Beijing, 100083, China; \\ ${ }^{\mathrm{b}}$ School of Mathematics, The University of Manchester, Oxford Road, Manchester, M13 9PL, UK.
}

\begin{abstract}
Modern medical studies show that chemotherapy can help most cancer patients, especially for those diagnosed early, to stabilize their disease conditions from months to years, which means the population of tumor cells remained nearly unchanged in quite a long time after fighting against immune system and drugs. In order to better understand the dynamics of tumor immune responses under chemotherapy, deterministic and stochastic differential equation models are constructed to characterize the dynamical change of tumor cells and immune cells in this paper. The basic dynamical properties, such as boundedness, existence and stability of equilibrium points, are investigated in the deterministic model. Extended stochastic models include stochastic differential equations (SDEs) model and continuous-time Markov chain (CTMC) model, which accounts for the variability in cellular reproduction, growth and death, interspecific competitions, and immune response to chemotherapy. The CTMC model is harnessed to estimate the extinction probability of tumor cells. Numerical simulations are performed, which confirms the obtained theoretical results.
\end{abstract}

Keywords Chemotherapy, Deterministic model, Stochastic model, Tumor

\section{Introduction}

Cancer treatment is a major public health problem in most parts of the world. It causes the highest mortality rate in economically developed countries and the second highest mortality rate in developing countries [1]. In 2012, there were about 14.1 million new cancer cases and 8.2 million deaths in the world based on the GLOBOCAN estimates. The occurrence of cancer is increasing as a result of population aging and growth, leading to an increasing prevalence of established risk factors such as smoking, overweight, physical inactivity, and changing reproductive patterns associated with urbanization and economic development [2].

The interactions between the immune system and growing tumor are quite complicated. Generally speaking, the immune response to tumor mainly involves the division and coordination of several kinds of immune cells. Once a tumor occurs in one tissue, B-lymphocytes products and secrets antibodies into the blood or places them on the surface of the tumor. In the meantime, the effector cells (Cytotoxic-T-Lymphocytes, CTLs) destroy the antigens constantly. This leads to T helper-lymphocytes, the antigen-bearing cells, to secret interleukins, which stimulates both $\mathrm{T}$ and $\mathrm{B}$ cells to divide. Finally, the immune response is terminated by T-suppressor cells. The process of immune response to a tumor seems unassailable. Unfortunately, the tumor processes a rigorous defense system that could interrupt the occur-

${ }^{*}$ Corresponding author.

Email address: jianxin.pan@manchester.ac.uk(J. Pan), xdliu@ustb.edu.cn(X. Liu) 
rence of a fully efficient response by preventing themselves from contacting with lymphocytes through the surface expression of ligands which initiate the apoptotic signal in the cytotoxic T-lymphocytes [3].

Accounting for the complexity of immune responses to a tumor, various mathematical models were developed to understand its dynamical mechanism in the past few decades. Among these models, most of them are deterministic (e.g., [4, 5, 6, 7, 8, 9, 10, 11, 12, 13, 14, 15, 16, 17, 18] and references therein), and only a few of them are stochastic ( e.g., [19, 20, 21, 22, 23] and references therein). In the investigation of deterministic models, some researchers focus on the estimation of parameters and the analysis of dynamical properties, such as stability, bifurcation and its stability and direction[4, 5, 6, 7, 8, 9, 10, 11], while others further extend the applications of these models by exploring the optimal control of cancer treatment $[12,13,14,15,16,17,18]$. Stochastic models are introduced to characterize immune responses to a tumor, which improves understanding of the pathological process from a different perspective. Sarkar and Banerjee [19] constructed a stochastic system, consisting of tumor cells, hunting predator cells and resting predator cells, and investigated the stochastic stability properties of the model. Albano and Giorno [20] proposed a stochastic model for solid tumor growth based on deterministic Gompertz law, and studied the effects of a time-dependent therapy via a numerical approach. Bose and Trimper [21] analyzed a stochastic model for tumor cell growth with both multiplicative and additive colored noises as well as nonzero cross correlations in between. Xu et al. [22] discussed the stochastic bifurcation for a tumor-immune system in the presence of a symmetric non-Gaussian Lévy noise, and found that stochastic dynamics induced by Gaussian and non-Gaussian Lévy noises were quite different. Kim et al. [23] constructed deterministic and stochastic models of cancer-virus dynamics, and investigated virus characteristic parameter sensitivities using a reproduction ratio. Overall, mathematical modelling plays an essential role in designing and analyzing clinical trials to oncology, which offers a potentially powerful tool in the development of improved treatment regimens.

Actually, many factors influence the effect of cancer treatment, such as the strength of patient's own immune response, the severity of the disease, and the application of the treatment. From the perspective of tumor therapy, chemotherapy is a very important treatment method which maybe cure part of patients with non-metastatic cancer. Even for patients with advanced cancer, it is still possible to extend their life spans to some extent. Taking lung cancer for example, it leads to approximately one third of all cancerrelated deaths, which is more than the aggregate proportion of breast, prostate, and colon cancer in each year. The median survival of patients with untreated metastatic non-small-cell lung cancer is only four to five months, with a survival rate at one year of only 10 percent [24]. However, many studies proved that chemotherapy, as a supportive care, slightly promotes the survival time for patients with advanced nonsmall-cell lung cancer [25, 26, 27]. Moreover, Cullen et al. [28] demonstrated that chemotherapy with the "best supportive care" reduced symptoms and improved the quality of life for lung cancer patients. Fortunately, a variety of new drugs have been found for treating metastatic non-small-cell lung cancer over the past two decades, such as the taxanes, gemcitabine, and vinorelbine. A number of phase II studies show that it has resulted in high response rates and prolonged survival at one year by combining one or more of these drugs with a platinum compound [29, 30, 31].

Inspired by the above researches, this paper attempts to explore the influence of chemotherapy 
on tumor-immune responses from a long-term perspective. Clinical medicine researches have shown that the stable period of a cancer patient with chemotherapy could remain for months or even a couple of years, which means that the population of tumor cells remained nearly unchanged in quite a long time after fighting against the immune system and drugs. Unfortunately, in most cases tumor cells eventually develop resistance to these chemicals and defeat immune system in vivo. However, it is still very significant to harness mathematical and statistical modeling to improve the understanding of the system dynamics of tumor-immune responses to chemotherapy, which should contribute to make some effective cancer treatment strategies. For this, we shall first construct a deterministic model by adding the terms of chemotherapy effect based on the immune responses system proposed in [32,33], and obtain asymptotically stable conditions of equilibriums. Moreover, considering much uncertainty in the process of treatment, such as the variability in cellular reproduction and death, the change of fight ability between immune system and tumor cell, and the fluctuation of chemotherapy effect, we extend the deterministic model to stochastic one, and further use a continuous-time Markov chain (CTMC) model to estimate the extinction probability of tumor cells.

The remaining sections are organized as follows. The deterministic ordinary differential equation (ODE) model that characterizes tumor-immune responses is constructed, and its basic dynamical properties are discussed in section 2. In section 3, a stochastic model is proposed based on the deterministic ODE model, and a continuous-time Markov chain model is employed to estimate the extinction probability of tumor cells. Some numerical simulations are performed to verify the theoretical results obtained in this paper. The paper ends with a brief conclusion.

\section{Deterministic mathematical model}

\subsection{The construction of ODE}

At time $t$, let $E(t)$ denote the population of effector immune cells (such as $\mathrm{CD} 8^{+} \mathrm{T}$ cells); $N(t)$ represents the population of normal cells; and $T(t)$ is the population of tumor cells. Pillis and Radunskaya $[32,33]$ proposed a mathematical model to describe the interactions of the tumor-immune system as follows:

$$
\left\{\begin{array}{l}
\frac{\mathrm{d} E}{\mathrm{~d} t}=s+\frac{\rho E T}{\alpha+T}-\beta_{1} E T-d_{1} E \\
\frac{\mathrm{d} N}{\mathrm{~d} t}=r_{1} N\left(1-b_{1} N\right)-\beta_{2} N T \\
\frac{\mathrm{d} T}{\mathrm{~d} t}=r_{2} T\left(1-b_{2} T\right)-\beta_{3} E T-\beta_{4} N T
\end{array}\right.
$$

with the initial conditions $E(0)>0, N(0)>0, T(0)>0$. In the first equation of system (1), the immune effector cells have a constant source $s$ and a constant death rate $d_{1}$. Effector cells are also recruited by tumor cells through a Michaelis-Menten term, $\frac{\rho E T}{\alpha+T}$, which serves to provide a saturation effect $[17,34,35]$. Here, both $\rho$ and $\alpha$ are positive constants. Furthermore, the competition between effector cells and tumor cells results in the death of two kinds of cells, which can be reflected by the terms $-\beta_{1} E T$ and $-\beta_{3} E T$. 
In the second and third equations of system (1), normal cells and tumor cells are produced by a logistic growth law. Parameters $r_{1}\left(r_{2}\right)$ and $b_{1}^{-1}\left(b_{2}^{-1}\right)$ are the maximal growth rate and the maximal carrying capacity of the biological environment for the normal cell population (tumor cell population), respectively. It's important to note that the parameter $r_{1}\left(r_{2}\right)$ incorporates both multiplication and death of the normal cell population (tumor cell population). Additionally, there are also competition between tumor and normal cells, which is described in the last terms of these two equations.

System (1) characterizes the immune system response to the tumor without therapy in vivo. When consideing the chemotherapy effect on the system, all the three cell populations would change through some mass-action dynamic behaviors. Naturally, chemotherapy has a different effect on each type cel1. By adding depletion term in each equation, a new deterministic model describing tumor-immune responses to chemotherapy is given by

$$
\left\{\begin{array}{l}
\frac{\mathrm{d} E}{\mathrm{~d} t}=s+\frac{\rho E T}{\alpha+T}-\beta_{1} E T-d_{1} E-c_{1} E \\
\frac{\mathrm{d} N}{\mathrm{~d} t}=r_{1} N\left(1-b_{1} N\right)-\beta_{2} N T-c_{2} N \\
\frac{\mathrm{d} T}{\mathrm{~d} t}=r_{2} T\left(1-b_{2} T\right)-\beta_{3} E T-\beta_{4} N T-c_{3} T,
\end{array}\right.
$$

with the initial conditions

$$
E(0)=E_{0}>0, N(0)=N_{0}>0, T(0)=T_{0}>0 .
$$

In system (2), the variables and parameters have the same meaning as what they are in system (1). Parameters $c_{1}, c_{2}$ and $c_{3}$ reflect the chemotherapy effect on the depletion rate of three types of cells. The growth rate of effector immune cells is greater than the natural death rate and clear rate by chemotherapy, and the growth rate of normal cells is larger than the clear rate by chemotherapy, so that a reasonable assumption is $\rho>c_{1}+d_{1}$ and $r_{1}>c_{2}$.

\subsection{Existence of equilibrium points}

In this section, we first prove that all solutions of the system (2) with initial conditions (3) are positive and ultimately bounded.

Suppose $E(t)$ is not always positive. Let $t_{1}$ be the first time such that $E\left(t_{1}\right)=0$, i.e., $t_{1}=\inf \{t \mid$ $t>0, E(t)=0\}$. Then we have $\frac{\mathrm{d} E\left(t_{1}\right)}{\mathrm{d} t}=s>0$ by the first equation of system (2), which means $E(t)<0$ for $t \in\left(t_{1}-\epsilon, t_{1}\right)$, where $\epsilon$ is an arbitrary small positive constant. This contradicts with the previous hypothesis, so that $E(t)$ is always positive under initial conditions (3).

Now, we shall show that both $N(t)$ and $T(t)$ are positive for all $t>0$ under initial conditions (3). By solving the second and third equation of system (3), we obtain

$$
\begin{aligned}
& N(t)=N(0) \exp \left\{\int_{0}^{t}\left[r_{1}\left(1-b_{1} N(s)\right)-\beta_{2} T(s)-c_{2}\right] \mathrm{d} s\right\}, \\
& T(t)=T(0) \exp \left\{\int_{0}^{t}\left[r_{2}\left(1-b_{2} T(s)\right)-\beta_{3} E(s)-\beta_{4} N(s)-c_{3}\right] \mathrm{d} s\right\} .
\end{aligned}
$$


Hence, it is easily to see that $N(t)>0$ and $T(t)>0$ for $t>0$ under initial conditions (3).

In the following, we analyze the boundedness of solutions of the system (2). By the first equation of system (2), we get

$$
\frac{\mathrm{d} E}{\mathrm{~d} t}=s+\frac{\rho E T}{\alpha+T}-\beta_{1} E T-d_{1} E-c_{1} E<s+\frac{\rho E T}{\alpha+T}-\left(d_{1}+c_{1}\right) E,
$$

Solving differential equation (4), we easily obtain

$$
E(t)<E(0) \mathrm{e}^{-\left(c_{1}+d_{1}\right) t}+\mathrm{e}^{-\left(c_{1}+d_{1}\right) t} \int_{0}^{t}\left[s+\frac{\rho E(u) T(u)}{\alpha+T(u)}\right] \mathrm{d} u .
$$

From the second equation of system (2), we have

$$
\frac{\mathrm{d} N}{\mathrm{~d} t}<r_{1} N\left(1-b_{1} N\right)-c_{2} N,
$$

which implies, by Kamke's comparison theorem,

$$
\lim _{x \rightarrow \infty} \sup N(t) \leq \frac{r_{1}-c_{2}}{b_{1} r_{1}},
$$

and

$$
N(t) \leq \max \left(N(0), \frac{r_{1}-c_{2}}{b_{1} r_{1}}\right) .
$$

Similarly, according to the third equation of system (2), we can arrive at the following inequality

$$
T(t) \leq \max \left(T(0), \frac{r_{2}-c_{3}}{b_{2} r_{2}}\right) .
$$

Based on the above analysis, we obtain the following theorem.

Theorem 1 For sufficiently large t, all solutions of system (2) with initial conditions (3) are positive and ultimately bounded.

After a period of treatment, cancer cells in the host may not increase, but keep in a stable state, i.e., the equilibrium point of the system. In order to solve the equilibrium points, the derivatives in left hand of system (2) are assumed to be equal to zero, so that the following equations are derived,

$$
\left\{\begin{array}{l}
s+\rho E T /(\alpha+T)-\beta_{1} E T-d_{1} E-c_{1} E=0, \\
r_{1} N\left(1-b_{1} N\right)-\beta_{2} N T-c_{2} N=0 \\
r_{2} T\left(1-b_{2} T\right)-\beta_{3} E T-\beta_{4} N T-c_{3} T=0 .
\end{array}\right.
$$

Clearly, if $T=0$, then system (2) has two disease-free equilibrium points $P_{0,1}^{*}=\left(E_{0,1}^{*}, N_{0,1}^{*}, T_{0}^{*}\right)=$ $\left(\frac{s}{c_{1}+d_{1}}, \frac{r_{1}-c_{2}}{b_{1} r_{1}}, 0\right)$ and $P_{0,2}^{*}=\left(E_{0,2}^{*}, N_{0,2}^{*}, T_{0}^{*}\right)=\left(\frac{s}{c_{1}+d_{1}}, 0,0\right)$. If $T \neq 0$, system (2) has two types of roots, that is,

$$
\begin{aligned}
& P_{1}^{*}=\left(E_{1}^{*}, N_{1}^{*}, T^{*}\right)=\left(\frac{s\left(\alpha+T^{*}\right)}{\left(\alpha+T^{*}\right)\left(\beta_{1} T^{*}+c_{1}+d_{1}\right)-\rho T^{*}}, \frac{r_{1}-c_{2}-\beta_{2} T^{*}}{b_{1} r_{1}}, T^{*}\right), \\
& P_{2}^{*}=\left(E_{2}^{*}, N_{2}^{*}, T^{*}\right)=\left(\frac{s\left(\alpha+T^{*}\right)}{\left(\alpha+T^{*}\right)\left(\beta_{1} T^{*}+c_{1}+d_{1}\right)-\rho T^{*}}, 0, T^{*}\right),
\end{aligned}
$$


where $T^{*}$ is one of the roots of equation (10), calculated by equation (9),

$$
f(T) \triangleq T^{3}+B T^{2}+C T+D=0,
$$

and

$$
\begin{aligned}
& B=\frac{b_{1} r_{1} r_{2}+c_{2} \beta_{4}-b_{1} c_{3} r_{1}-\beta_{4} r_{1}}{\beta_{2} \beta_{4}-b_{1} b_{2} r_{1} r_{2}}+\frac{c_{1}+d_{1}+\alpha \beta_{1}-\rho}{\beta_{1}}, \\
& C=\frac{\left(c_{1}+d_{1}+\alpha \beta_{1}-\rho\right)\left(b_{1} r_{1} r_{2}+c_{2} \beta_{4}-b_{1} c_{3} r_{1}-\beta_{4} r_{1}\right)}{\beta_{1}\left(\beta_{2} \beta_{4}-b_{1} b_{2} r_{1} r_{2}\right)}+\frac{\alpha\left(c_{1}+d_{1}\right)}{\beta_{1}}-\frac{b_{1} r_{1} s \beta_{3}}{\beta_{1}\left(\beta_{2} \beta_{4}-b_{1} b_{2} r_{1} r_{2}\right)}, \\
& D=\frac{\alpha\left(c_{1}+d_{1}\right)\left(b_{1} r_{1} r_{2}+c_{2} \beta_{4}-b_{1} c_{3} r_{1}-\beta_{4} r_{1}\right)-b_{1} r_{1} s \alpha \beta_{3}}{\beta_{1}\left(\beta_{2} \beta_{4}-b_{1} b_{2} r_{1} r_{2}\right)} .
\end{aligned}
$$

The root discriminant of equation (10) is given by

$$
\Delta=\frac{1}{4}\left[f\left(\frac{-B}{3}\right)\right]^{2}+\frac{1}{9}\left[f^{\prime}\left(\frac{-B}{3}\right)\right]^{3} .
$$

So equation (10) has one double or triple roots if $\Delta=0$; equation (10) has one real root and two conjugate complex roots if $\Delta>0$; equation (10) has three different real roots if $\Delta<0$.

In order to analyze the situation of roots for equation (10) by Descartes' guidelines, we first give some assumed conditions.

$\left(H_{1}\right)$ any of the following conditions: $\left(a_{1}\right) B \geq 0, D<0 ;\left(a_{2}\right) C \leq 0, D<0 ;\left(a_{3}\right) B<0, C>$ $0, D<0, \Delta>0 ;\left(a_{4}\right) B<0, C=0, D=0 ;\left(a_{5}\right) C<0, D=0$;

$\left(H_{2}\right)$ any of the following conditions: $\left(b_{1}\right) B<0, C>0, D>0, \Delta<0 ;\left(b_{2}\right) B<0, C>0, D=$ $0, B^{2}>4 C$;

$\left(H_{3}\right) B<0, C>0, D<0, \Delta<0$;

$\left(H_{4}\right)$ any of the following conditions: $\left(c_{1}\right) B \geq 0, C \geq 0, D \geq 0 ;\left(c_{2}\right) D>0, \Delta>0$;

Then we easily obtain the following lemma based on Descartes' rule.

Lemma 1 For equation (10), we have the following conclusions.

(i) If $\left(H_{1}\right)$ holds, then equation (10) has the only positive real root;

(ii) If $\left(\mathrm{H}_{2}\right)$ holds, then equation (10) has two different positive real roots;

(iii) If $\left(H_{3}\right)$ holds, then equation (10) has three different positive real roots;

(iv) If $\left(H_{4}\right)$ holds, then equation (10) has no positive real roots.

Consequently, it is not difficult to find that system (2) has six epidemic equilibrium points at most. We write them by

$$
P_{i, 1}^{*}=\left(E_{i, 1}^{*}, N_{i, 1}^{*}, T_{i}^{*}\right), P_{i, 2}^{*}=\left(E_{i, 2}^{*}, 0, T_{i}^{*}\right), i=1,2,3 .
$$

However, the population of normal cells is greater than zero in host, so in the next section we only investigate the dynamical properties of disease-free equilibrium point $P_{0,1}^{*}=\left(E_{0,1}^{*}, N_{0,1}^{*}, 0\right)$ and the positive equilibrium points $P_{i, 1}^{*}=\left(E_{i, 1}^{*}, N_{i, 1}^{*}, T_{i}^{*}\right), i=1,2,3$. 


\subsection{Stability of equilibrium points}

In this section, we shall discuss the stability of system (2) at its disease-free steady state and the positive steady states.

Let $P_{i, 1}^{*}=\left(E_{i, 1}^{*}, N_{i, 1}^{*}, T_{i}^{*}\right)(i=0,1,2,3)$ be any arbitrary steady state, then the Jacobian matrix $\mathbf{J}$ of system (2) about $P_{i, 1}^{*}$ is given by

$$
\mathbf{J}=\left[\begin{array}{ccc}
\frac{\rho T_{i}^{*}}{\alpha+T_{i}^{*}}-\beta_{1} T_{i}^{*}-c_{1}-d_{1} & 0 & \frac{\alpha \rho E_{i, 1}^{*}}{\left(\alpha+T_{i}^{*}\right)^{2}} \\
0 & -b_{1} r_{1} N_{i, 1}^{*} & \beta_{2} N_{i, 1}^{*} \\
-\beta_{3} T_{i}^{*} & -\beta_{4} T_{i}^{*} & r_{2}-c_{3}-2 b_{2} r_{2} T_{i}^{*}-\beta_{3} E_{i, 1}^{*}-\beta_{4} N_{i, 1}^{*}
\end{array}\right],
$$

and the corresponding characteristic equation can be written as

$$
|\lambda \mathbf{I}-\mathbf{J}|=0,
$$

where $\mathbf{I}$ is a unit matrix. For the steady state $P_{0,1}^{*}=\left(E_{0,1}^{*}, N_{0,1}^{*}, 0\right)$, equation (11) reduces to

$$
\left(\lambda+c_{1}+d_{1}\right)\left(\lambda+r_{1}-c_{2}\right)\left[\lambda-\left(r_{2}-c_{3}\right)+\frac{\beta_{3} s}{c_{1}+d_{1}}+\frac{\beta_{4}\left(r_{1}-c_{2}\right)}{b_{1} r_{1}}\right]=0 .
$$

Hence, according to the previous assumption $r_{1}>c_{2}$, we have the following theorem by Routh-Hurwitz Criterion.

Theorem 2 If there exists a disease-free equilibrium point $P_{0,1}^{*}$ and $r_{2}-c_{3}-\frac{\beta_{3} s}{c_{1}+d_{1}}-\frac{\beta_{4}\left(r_{1}-c_{2}\right)}{b_{1} r_{1}}<0$, then $P_{0,1}^{*}$ is locally asymptotically stable.

For any positive steady state $P_{i, 1}^{*}=\left(E_{i, 1}^{*}, N_{i, 1}^{*}, T_{i}^{*}\right), i=1,2,3$, equation (11) reduces to

$$
\lambda^{3}+A_{1} \lambda^{2}+A_{2} \lambda+A_{3}=0
$$

where

$$
\begin{aligned}
& A_{1}=\frac{s}{E_{i, 1}^{*}}+b_{1} r_{1} N_{i, 1}^{*}+b_{2} r_{2} T_{i}^{*}>0, \\
& A_{2}=b_{1} b_{2} r_{1} r_{2} N_{i, 1}^{*} T_{i}^{*}+\beta_{2} \beta_{4} T_{i}^{*} N_{i, 1}^{*}+\frac{s\left(r_{1} b_{1} N_{i, 1}^{*}+r_{2} b_{2} T_{i}^{*}\right)}{E_{i, 1}^{*}}+\frac{\alpha \beta_{3} \rho E_{i, 1}^{*} T_{i}^{*}}{\left(\alpha+T_{i}^{*}\right)^{2}}>0, \\
& A_{3}=\frac{s\left(b_{1} b_{2} r_{1} r_{2} N_{i, 1}^{*} T_{i}^{*}+\beta_{2} \beta_{4} T_{i}^{*} N_{i, 1}^{*}\right)}{E_{i, 1}^{*}}+\frac{b_{1} r_{1} \alpha \beta_{3} \rho E_{i, 1}^{*} N_{i, 1}^{*} T_{i}^{*}}{\left(\alpha+T_{i}^{*}\right)^{2}}>0 .
\end{aligned}
$$

Thus, by directly calculating, we easily get

$$
\begin{aligned}
A_{1} A_{2}-A_{3} & =\frac{s}{E_{i, 1}^{*}}\left(\frac{s\left(b_{1} r_{1} N_{i, 1}^{*}+b_{2} r_{2} T_{i}^{*}\right)}{E_{i, 1}^{*}}+\frac{\alpha \beta_{3} \rho E_{i, 1}^{*} T_{i}^{*}}{\left(\alpha+T_{i}^{*}\right)^{2}}\right)+\frac{b_{2} r_{2} \alpha \beta_{3} \rho E_{i, 1}^{*}\left(T_{i}^{*}\right)^{2}}{\left(\alpha+T_{i}^{*}\right)^{2}} \\
& +\left(b_{1} r_{1} N_{i, 1}^{*}+r_{2} b_{2} T_{i}^{*}\right)\left(b_{1} b_{2} r_{1} r_{2} N_{i, 1}^{*} T_{i}^{*}+\beta_{2} \beta_{4} T_{i}^{*} N_{i, 1}^{*}+\frac{s\left(b_{1} r_{1} N_{i, 1}^{*}+b_{2} r_{2} T_{i}^{*}\right)}{E_{i, 1}^{*}}\right) \\
& >0 .
\end{aligned}
$$

Similarly, we have the following theorem by the application of Routh-Hurwitz Criterion. 
Theorem 3 If there exists a positive steady state $P_{i, 1}^{*}=\left(E_{i, 1}^{*}, N_{i, 1}^{*}, T_{i}^{*}\right)(i=1,2,3)$ in system (2), then it is locally asymptotically stable.

Note that system (2) possesses three positive equilibrium points at most. However, the existence of the equilibrium point is a stronger condition. As long as the positive steady state exists, it must be locally asymptotically stable.

\section{Stochastic tumor model}

\subsection{The construction of CTMC model}

According to the analysis of deterministic model, we can distinguish the tumor model into 9 stages, and a Continuous Time Markov Chain (CTMC) model can be derived based on the branching processes theory [36]. Setting $\vec{X}(t)=(E, N, T)^{t r}$ be a continuous random vector and $t \in[0, \infty)$. A state change in short time $\Delta t$ is $\Delta \vec{X}=\vec{X}(t+\Delta t)-\vec{X}$. Based on the deterministic model, we propose 9 possible state changes in the CTMC model and their corresponding occurrence rates as shown in table 1.

Table 1: State changes and their associated occurrence rates during $\Delta t$

\begin{tabular}{lccl}
\hline i & $\Delta \vec{X}^{t r}$ & $P_{i} \Delta t+o(\Delta t)$ & Description \\
\hline 1 & $(1,0,0)$ & $\left(s+\frac{\rho E T}{\alpha+T}\right) \Delta t+o(\Delta t)$ & Reproduction of a immune effect cell and the recruit by tumor cells. \\
2 & $\left(-\beta_{1}, 0,-\beta_{3}\right)$ & $(E T) \Delta t+o(\Delta t)$ & The competition between immune effect cells and tumor cells. \\
3 & $(-1,0,0)$ & $\left(d_{1} E+c_{1} E\right) \Delta t+o(\Delta t)$ & Natural death and response to chemotherapy of immune effect cells. \\
4 & $(0,1,0)$ & $r_{1} N\left(1-b_{1} N\right) \Delta t+o(\Delta t)$ & Logistic growth of normal cells. \\
5 & $\left(0,-\beta_{2},-\beta_{4}\right)$ & $(N T) \Delta t+o(\Delta t)$ & The competition between normal cells and tumor cells. \\
6 & $(0,-1,0)$ & $\left(c_{2} N\right) \Delta t+o(\Delta t)$ & Response to chemotherapy of normal cells. \\
7 & $(0,0,1)$ & $r_{2} T\left(1-b_{2} T\right) \Delta t+o(\Delta t)$ & Logistic growth of tumor cells. \\
8 & $(0,0,-1)$ & $\left(c_{3} T\right) \Delta t+o(\Delta t)$ & Response to chemotherapy of tumor cells. \\
9 & $(0,0,0)$ & $1-\sum_{i=1}^{8} P_{i} \Delta t+o(\Delta t)$ & No change. \\
\hline
\end{tabular}

In the state change 1 of table 1 , the $\Delta \vec{X}^{t r}=(1,0,0)$ represents that the number of effector immune cell increases "1" with no change of normal cell and tumor cell. The state change only happens in reproduction of effector immune cell and also the recruit by tumor cell, the corresponding occurrence rates are $(s \Delta t+o(\Delta t))$ and $\left(\frac{\rho E T}{\alpha+T} \Delta t+o(\Delta t)\right)$, separately, and $o(\Delta t)$ represents a error term of occurrence rate in the process of state change. For the competition between immune effect cell and tumor cell, $\beta_{1}$ of immune effect cells and $\beta_{3}$ of tumor cells would dead with a occurrence rate $((E T) \Delta t+o(\Delta t))$. Similar competition happens between normal cell and tumor cell with cell lose $\Delta \vec{X}^{t r}=\left(0,-\beta_{2},-\beta_{4}\right)$ and corresponding occurrence rate $((N T) \Delta t+o(\Delta t))$. Those are state changes 2 and 5 . In state change 
3 , the $\Delta \vec{X}^{t r}=(-1,0,0)$ implies a death of immune effect cell, this can be caused by nature death or response to chemotherapy of immune effect cell, with corresponding occurrence rates $\left(d_{1} E \Delta t+o(\Delta t)\right)$ and $\left(c_{1} E \Delta t+o(\Delta t)\right)$ respectively. Similar logic and analysis can be conducted to normal cells and tumor cells. Then eventually we will have those 8 state changes and their associated occurrence rates in table 1.

Based on the state changes and their corresponding occurrence rates in table 1, we can build a Itô process by computing the expectations $\mathbb{E}(\Delta \vec{X})$ and $\mathbb{E}\left(\Delta \vec{X} \Delta \vec{X}{ }^{t r}\right)$. Here, the occurrence rates are approximately viewed as the corresponding probabilities for each state change. Therefore, neglecting terms higher than $o(\Delta t)$, the expectation $\mathbb{E}(\Delta \vec{X})$ yields in the following form

$$
\mathbb{E}(\Delta \vec{X})=\sum_{i=1}^{8} P_{i} \Delta t \Delta \vec{X}_{i}=\vec{f}(E, N, T) \Delta t
$$

and

$$
\vec{f}=\left(\begin{array}{l}
s+\frac{\rho E T}{\alpha+T}-\beta_{1} E T-d_{1} E-c_{1} E \\
r_{1} N\left(1-b_{1} N\right)-\beta_{2} N T-c_{2} N \\
r_{2} T\left(1-b_{2} T\right)-\beta_{3} E T-\beta_{4} N T-c_{3} T
\end{array}\right)
$$

We can treat this as a mean part, without randomness. By adding up some Weiner processes, we obtain the expression of an approximate covariance matrix of $\Delta \vec{X}$ as follows

$$
\mathbb{E}\left(\Delta \vec{X} \Delta \vec{X}^{t r}\right) \approx \sum_{i=1}^{8} P_{i} \Delta t \Delta \vec{X}_{i} \Delta \vec{X}_{i}^{t r}=\Sigma(E, N, T) \Delta t
$$

where the diffusion matrix $\Sigma$ is

$$
\left(\begin{array}{ccc}
P_{1}+\beta_{1}^{2} P_{2}+P_{3} & 0 & \beta_{1} \beta_{3} P_{2} \\
0 & P_{4}+\beta_{2}^{2} P_{5}+P_{6} & \beta_{2} \beta_{4} P_{5} \\
\beta_{1} \beta_{3} P_{2} & \beta_{2} \beta_{4} P_{5} & \beta_{3}^{2} P_{2}+\beta_{4}^{2} P_{5}+P_{7}+P_{8}
\end{array}\right) .
$$

Due to the difficulty of finding the square root of the diffusion matrix $\Sigma$ in practice, we use an equivalent matrix $G$, which also full fills $G G^{t r}=\Sigma$. And elements in $G$ are

$$
g_{j, i}=X_{i, j} P_{i}^{1 / 2}
$$

where $X_{i, j}$ is the $j$-th element in $\vec{X}_{i}$ [37]. Then we could have

$$
G=\left(\begin{array}{cccccccc}
P_{1}^{1 / 2} & -\beta_{1} P_{2}^{1 / 2} & -P_{3}^{1 / 2} & 0 & 0 & 0 & 0 & 0 \\
0 & 0 & 0 & P_{4}^{1 / 2} & -\beta_{2} P_{5}^{1 / 2} & -P_{6}^{1 / 2} & 0 & 0 \\
0 & -\beta_{3} P_{2}^{1 / 2} & 0 & 0 & -\beta_{4} P_{5}^{1 / 2} & 0 & P_{7}^{1 / 2} & P_{8}^{1 / 2}
\end{array}\right)
$$


Therefore, the stochastic deferential equation for tumor immune response process has the form as below

$$
\left\{\begin{array}{l}
\mathrm{d} \vec{X}(t)=\vec{f}(E, N, T) d t+G(E, N, T) \mathrm{d} \vec{W}(t), \\
\vec{X}(0)=(E(0), N(0), T(0)),
\end{array}\right.
$$

where $\vec{W}(t)=\left(W_{1}(t), W_{2}(t), \ldots, W_{8}(t)\right)^{t r}$ is a vector of eight independent Wiener process, and $\mathrm{d} \vec{W}_{i}(t)$ is a Gaussian increment with mean 0 and identity matrix I as covariance. This SDE happens to be in an Itô process. Specifically, there is no need for chemotherapy treatment if no tumor cell. In this case, we have $E(0)=\bar{E}, N(0)=\bar{N}$, and $T(0)=0$, with $c_{1}=c_{2}=c_{3}=0$. Therefore, the Itô process can be simplified as below

$$
\left\{\begin{array}{l}
\mathrm{d} E=\left(s-d_{1} E\right) d t+\sqrt{s} \mathrm{~d} W_{1}-\sqrt{d_{1} E} \mathrm{~d} W_{3} \\
\mathrm{~d} N=\left[r_{1} N\left(1-b_{1} N\right)\right] \mathrm{d} t+\sqrt{r_{1} N\left(1-b_{1} N\right)} \mathrm{d} W_{4} .
\end{array}\right.
$$

Clearly, equation (15) is a closed scaler system. However, it is quite difficult to calculate its mean and variance for all cases because of multiplicative noises. Note that tumor patients may have a period of stability after receiving chemotherapy, which indicates that model (15) may be globally stable under some conditions, and that random factors cannot fundamentally change the stability of model (15) in some cases. Thus, we further give the following assumptions.

$\left(H_{5}\right)$ There exists a set $\Phi$ such that stochastic model (15) is globally stable for $(E, N, T) \in \Phi$.

$\left(H_{6}\right)$ For any function $f(E, N, T),(E, N, T) \in \Phi, \int_{0}^{t} f(E, N, T) \mathrm{d} W_{i}(u)$ is martingale $(i=$ $1,2, \cdots, 8)$.

Under such assumptions, we can solve the mean and variance under above conditions by applying Itô's formula [38]. From the first equation of equations (15) we get

$$
E(t)=E(0)+\int_{0}^{t}\left(s-d_{1} E(u)\right) \mathrm{d} u+\int_{0}^{t} \sqrt{s} \mathrm{~d} W_{1}(u)-\int_{0}^{t} \sqrt{d_{1} E(u)} \mathrm{d} W_{3}(u) .
$$

Because $\int_{0}^{t} \sqrt{s} \mathrm{~d} W_{1}(u)$ and $\int_{0}^{t} \sqrt{d_{1} E(u)} \mathrm{d} W_{3}(u)$ are both martingales, we have

$$
\mathbb{E}\left[\int_{0}^{t} \sqrt{s} \mathrm{~d} W_{1}(u)\right]=0, \quad \mathbb{E}\left[\int_{0}^{t} \sqrt{d_{1} E(u)} \mathrm{d} W_{3}(u)\right]=0 .
$$

Hence, by equation (16) we obtain

$$
\mathbb{E}(E(t))=\mathbb{E}(E(0))+\int_{0}^{t}\left(s-d_{1} \mathbb{E}(E(u))\right) \mathrm{d} u .
$$

Since the system described by equation (15) is in the stable state and assume $E(0)=\bar{E}$ which means the expectation $\mathbb{E}(E(t))=\mathbb{E}(E(0))=\bar{E}$ for all $t \geq 0$, thus we know that in equation (17)

$$
s-d_{1} \mathbb{E}(E(t))=0 \text {, i.e. } \mathbb{E}(E(t))=\bar{E}=\frac{s}{d_{1}} \text { for all } t \geq 0 .
$$


In the following we use the Itô formula to calculate the variance of $E(t)$. By Ito formula,

$$
\begin{aligned}
\mathrm{d} E^{2}(t)= & 2 E(t) \mathrm{d} E(t)+(\mathrm{d} E(t))^{2} \\
= & 2 E(t)\left[\left(s-d_{1} E(t)\right) \mathrm{d} t+\sqrt{s} \mathrm{~d} W_{1}(t)-\sqrt{d_{1} E(t)} \mathrm{d} W_{3}(t)\right] \\
& +\left[\left(s-d_{1} E(t)\right) \mathrm{d} t+\sqrt{s} \mathrm{~d} W_{1}(t)-\sqrt{d_{1} E(t)} \mathrm{d} W_{3}(t)\right]^{2} \\
= & \left(s+\left(d_{1}+2 s\right) E(t)-2 d_{1} E^{2}(t)\right) \mathrm{d} t+2 E(t) \sqrt{s} \mathrm{~d} W_{1}(t)-2 E(t) \sqrt{d_{1} E(t)} \mathrm{d} W_{3}(t),
\end{aligned}
$$

where

$$
(\mathrm{d} t)^{2}=\mathrm{d} W_{1}(t) \mathrm{d} W_{3}(t)=\mathrm{d} t \mathrm{~d} W_{1}(t)=\mathrm{d} t \mathrm{~d} W_{3}(t)=0, \quad\left(\mathrm{~d} W_{1}(t)\right)^{2}=\left(\mathrm{d} W_{3}(t)\right)^{2}=\mathrm{d} t .
$$

Similar to the calculus for the equation (17) and by equation (18) we get

$$
\begin{aligned}
\mathbb{E}\left[E^{2}(t)\right] & =\mathbb{E}\left[E^{2}(0)\right]+\int_{0}^{t}\left[s+\left(d_{1}+2 s\right) \mathbb{E}[E(u)]-2 d_{1} \mathbb{E}\left[E^{2}(u)\right]\right] \mathrm{d} u \\
& =\mathbb{E}\left[E^{2}(0)\right]+\int_{0}^{t}\left[2 s(1+\bar{E})-2 d_{1} \mathbb{E}\left(E^{2}(u)\right)\right] \mathrm{d} u,
\end{aligned}
$$

Thus, we further obtain

$$
\left\{\begin{array}{l}
\frac{\mathrm{d} \mathbb{E}\left[E^{2}(t)\right]}{d t}+2 d_{1} \mathbb{E}\left[E^{2}(t)\right]=2 s(1+\bar{E}), \\
\mathbb{E}\left[E^{2}(0)\right]=\bar{E}^{2}=\left(\frac{s}{d_{1}}\right)^{2} .
\end{array}\right.
$$

Then we have $\mathbb{E}\left[E^{2}(t)\right]=\bar{E}+\bar{E}^{2}-\bar{E} \mathrm{e}^{-\frac{2 s t}{\bar{E}}}$. Hence,

$$
\operatorname{Var}[E(t)]=\mathbb{E}\left[E^{2}(t)\right]-[\mathbb{E}[E(t)]]^{2}=\bar{E}+\bar{E}^{2}-\bar{E} \mathrm{e}^{-\frac{2 s t}{\bar{E}}}-\bar{E}^{2}=\bar{E}\left(1-\mathrm{e}^{-\frac{2 s t}{\bar{E}}}\right) .
$$

That is to say, when $t \gg 1$, it holds that $\operatorname{Var}[E(t)] \approx \bar{E}$.

For the second equation in equations (15), similarly we get

$$
\mathbb{E}(N(t))=\mathbb{E}(N(0))+\int_{0}^{t}\left[r_{1} \mathbb{E}(N(u))-r_{1} b_{1} \mathbb{E}\left(N^{2}(u)\right)\right] \mathrm{d} u .
$$

If we assume $\mathbb{E}(N(t))$ is independent of $t$ and $N(0)=\bar{N}$, then

$$
r_{1} \mathbb{E}(N(t))-r_{1} b_{1} \mathbb{E}\left(N^{2}(t)\right)=0 \text {, i.e. } \mathbb{E} N^{2}(t)=\frac{\mathbb{E} N(t)}{b_{1}}, \text { for all } t \geq 0 .
$$

Thus,

$$
\operatorname{Var}(N(t))=\mathbb{E} N^{2}(t)-[\mathbb{E} N(t)]^{2}=\frac{\mathbb{E} N(t)}{b_{1}}-[\mathbb{E} N(t)]^{2}=\frac{\bar{N}}{b_{1}}-\bar{N}^{2}
$$

\subsection{Extinction probability of tumor cell}

Based on the infinitesimal probabilities ( $\approx$ occurrence rates) for state-changes in table 1 , a timehomogeneous Continued Time Markov Chain (CTMC) model can be formulated, which means that the probability of change of states only depends on the current state. After an initial existence of tumor cell, both normal cell and effect cell are assumed to be and will remain at the steady state, i.e., $E(t)=\bar{E}$ 
and $N(t)=\bar{N}$. Assuming the effect of chemotherapy and competitions among tumor cells exist and each tumor cell acts independently while again the dynamics do not depend on history, so we only need to concentrate on the CTMC of $T(t)$. The backward Kolmogorov equation can give the probability of a CTMC system that will be in a given subset of states. So under the simplification of all assumptions above, we can solve the extinction probability of tumor cells by using the backward Kolmogorov equation. According to the theory from the branching process, the steady-state solution of the backward Kolmogorov equation is equivalent to the extinction probability, i.e., $\lim _{t \rightarrow \infty} T(t)=0$ [40].

Next, we use the Probability Generating Function (p.g.f) of $T(t)$ to build the backward Kolmogorov equation. Setting $T(0)=1$, then the p.g.f of $T(t)$ is

$$
\phi(t ; x)=\sum_{k} \operatorname{Pr}\{T(t)=k \mid T(0)=1\} x^{k} .
$$

Hence, the backward Kolmogorov differential equation for $\phi(t, x)$ can be written as [41]

$$
\frac{\partial \phi}{\partial t}=g(x)=\sum_{k=0}^{\infty} \frac{\partial \operatorname{Pr}\{T(t)=k \mid T(0)=1\}}{\partial t} x^{k} .
$$

Therefore, the infinitesimal probabilities in table (1) and equation

$$
\operatorname{Pr}\{T(t)=k \mid T(0)=1\} \approx I_{1, k}+a_{k} \Delta t+o(\Delta t),
$$

can be employed to estimate $\operatorname{Pro}\{T(t)=k \mid T(0)=1\}$, where $I_{1, k}=1$ if $k=1$, otherwise $I_{1, k}=0$. $a_{k}$ is the infinitesimal probability of state change $(\Delta E, \Delta N, k-1)$, which can make the tumor cell change from 1 to $k$. Based on the whole probability $\sum_{k=0}^{\infty} \operatorname{Pr}\{T(t)=k \mid T(0)=1\}=1$ and equation (25), it is easy to get $\sum_{k=0}^{\infty} a_{k}=0$. Then we have the backward Kolmogorov differential equation as follows

$$
\frac{\partial \phi}{\partial t}=g(x)=\sum_{k=0}^{\infty} a_{k} x^{k} .
$$

Set the backward Kolmogorov differential equation (26) equal to zero, and the root of that equation can be used to estimate the extinction probability of $\operatorname{Pr}\{T(t)=0 \mid T(0)=1\}$, which satisfies the following lemma [41, 42].

Lemma 2 Assume that $\tilde{p}$ is the minimum root of the backward Kolmogorov differential equation (26). If $\tilde{p} \in[0,1)$, then $\tilde{p}$ is the approximate ultimate extinction probability.

In the following, we shall discuss the extinction probability by the application of lemma 2 . Because $\sum_{k=0}^{\infty} a_{k}=0$, it is easy to see that $p=1$ is always a solution of $g(p)=0$. In practice, there may exist another solution $0 \leq \tilde{p} \leq 1$. However, before applying the lemma to the model, three assumptions need to be satisfied first: i) the state changes describing in table 1 are independent, ii) the initial state of the model is $\vec{X}(0)=(E(0)=\bar{E}, N(0)=\bar{N}, T(0)=m)^{t r}$, iii) $\tilde{p}$ exists. According to the Galton Watson branching theory, the ultimate extinction probability of tumor cells $\operatorname{Pr}\left\{\lim _{t \rightarrow \infty} T(t)=0\right\}$ for our CTMC 
model can be written as

$$
\operatorname{Pr}\left\{\lim _{t \rightarrow \infty} T(t)=0 \mid E(0)=\bar{E}, N(0)=\bar{N}, T(0)=m\right\}=\tilde{p}^{m} .
$$

Under our SDE model, since it is time-homogeneous we are only interested in the change of state during $\Delta t$. In a short period of time $\Delta t$ and with initial tumor cell count 1 , all changes of the state are limited in: i) start with 1 tumor cell and die out during $\Delta t$, i.e., $\operatorname{Pr}\{T(t)=0 \mid T(0)=1\} \approx 0+a_{0} \Delta t+o(\Delta t)$; ii) start with 1 tumor cell and duplicate into 2 tumor cell during $\Delta t$, i.e., $\operatorname{Pr}\{T(t)=2 \mid T(0)=$ $1\} \approx 0+a_{2} \Delta t+o(\Delta t)$; iii) start with 1 tumor cell and end with 1 tumor cell during $\Delta t$, i.e., $\operatorname{Pr}\{T(t)=1 \mid T(0)=1\} \approx 1+a_{1} \Delta t+o(\Delta t)$. Assume that each change of state obeys uniform distribution. For example, $P_{5}$ is the probability of $-\beta_{4}$ tumor cell due to the competition between normal cell and tumor cell, while $P_{5} / \beta_{4}$ is the probability of -1 tumor cell due to the same reason. According to the infinitesimal probabilities in table (1), with setting $T=1$ we have

$$
\left\{\begin{array}{l}
a_{0}=\frac{1}{\beta_{3}} P_{2}+\frac{1}{\beta_{4}} P_{5}+P_{8}=\frac{1}{\beta_{3}} \bar{E}+\frac{1}{\beta_{4}} \bar{N}+c_{3}, \\
a_{1}=-\left\{a_{0}+a_{2}\right\}=-\left\{\frac{1}{\beta_{3}} \bar{E}+\frac{1}{\beta_{4}} \bar{N}+c_{3}+r_{2}\left(1-b_{2}\right)\right\}, \\
a_{2}=P_{7}=r_{2}\left(1-b_{2}\right) .
\end{array}\right.
$$

Then $g(p)=0$ will be

$$
g(p)=a_{0}+a_{1} p+a_{2} p^{2}=0,
$$

where $a_{1}^{2}-4 a_{0} a_{2} \geq 0$ always holds. It is easy to see that equation (29) always has a solution $p=1$. Based on the definition of $a_{i}(i=1,2,3)$, the minimum solution of equation (29) $\tilde{p}$ can be achieved as follows

$$
\tilde{p}=\frac{\bar{E} / \beta_{3}+\bar{N} / \beta_{4}+c_{3}}{r_{2}\left(1-b_{2}\right)} .
$$

Specifically, ignoring the competition among $E, N$ and $T$, we easily get

$$
\left\{\begin{array}{l}
a_{0}=c_{3} \\
a_{1}=-\left(c_{3}+r_{2}\left(1-b_{2}\right)\right) \\
a_{2}=r_{2}\left(1-b_{2}\right)
\end{array}\right.
$$

so the extinction probability of tumor cell is

$$
\tilde{p}=\frac{c_{3}}{r_{2}\left(1-b_{2}\right)} .
$$

Consequently, the following theorem can be obtained. 
Theorem 4 Assume that $0 \leq \tilde{p}<1$ is the minimum solution of $g(p)=0$. If $\left(H_{1}\right),\left(H_{5}\right)$ and $\left(H_{6}\right)$ hold and $\tilde{p}$ exists, then system (2) has an unique positive equilibrium point and the ultimate extinction probability of tumor cells with initial state $\vec{X}(0)=(E(0)=\bar{E}, N(0)=\bar{N}, T(0)=m)^{t r}$ is

$$
\operatorname{Pr}\left\{\lim _{t \rightarrow \infty} T(t)=0 \mid E(0)=\bar{E}, N(0)=\bar{N}, T(0)=m\right\}=\tilde{p}^{m} .
$$

Moreover, if the disease-free equilibrium exists, the extinction probability of tumor cells would approximately be 1 .

\section{Numerical simulations and discussions}

In this section, some numerical simulations are performed to verify the main results obtained above.

The effect of chemotherapy on the clearance of tumor cells is the key to success in cancer treatment, so we focus on how the change of parameter $c_{3}$ affects the stability of system (2). In order to reveal the role of parameter $c_{3}$ in cancer treatment, we only change the value of $c_{3}$, and remain the same values for the other parameters in system (2). All the value of parameters in system (2) are shown in the columns of 'Data 1' and 'Data 2' in table 2. The choices of the parameter values can be referred to literature $[9,17,32,35,43,44,45]$, where the values are got from clinical trials.

When parameters values are taken from the column of 'Data 1', the phase figure and time figure of system (2) are presented in Fig. 1. In this case, system (2) has an unique positive equilibrium point which should be locally asymptotically stable according to theorem 3 . Figure (a) displays that all trajectories tend to the positive equilibrium point $P_{i, 1}^{*}$, and figure (b) shows that the number of three types of cells remains unchanged after a period of fluctuation. It implies that the quantity of tumor cells can be controlled in a very small range with the help of chemotherapy, which offers a reasonable explanation for why tumor patients could maintain a stable condition from months to years after receiving chemotherapy.

(a)

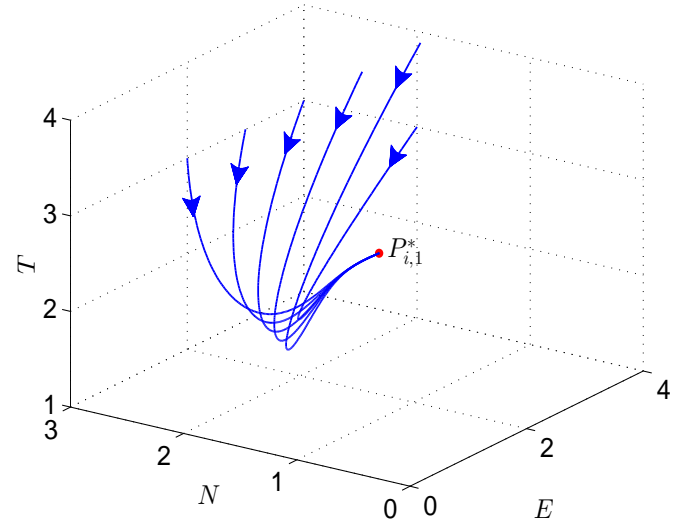

(b)

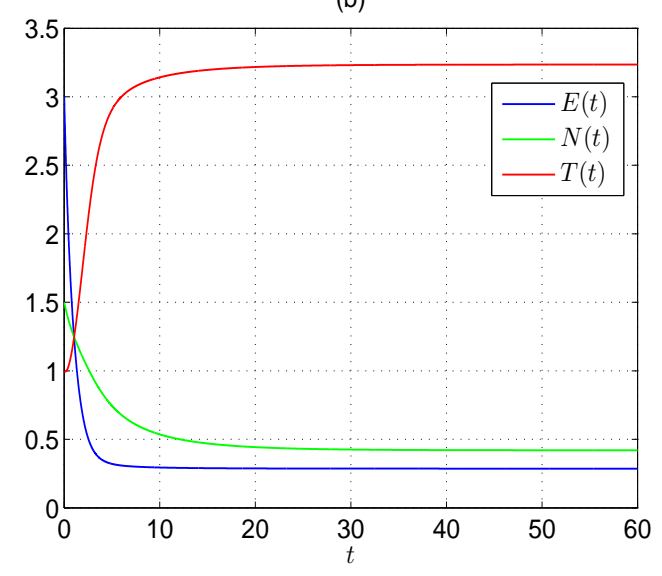

Fig.1. The unique infected equilibrium $P_{i, 1}^{*}=(4.29,1.53,0.057)$ is locally asymptotically stable. In figure (a), the initial values are $(3,3,3),(2,3,3),(4,3,3),(5,3,3),(6,3,3),(4,2,3)$, respectively. The initial value is $(3,1.5,1)$ in figure (b).

In the following, taking the parameter values from the column of 'Data 2', we obtain the phase figure and time figure of system (2) as shown in Fig.2. Under these parameter values, system (2) has 
Table 2: Parameter Values

\begin{tabular}{|c|c|c|c|c|}
\hline Parameters & Definition & Unit & Data 1 & Data 2 \\
\hline$s$ & Source rate of the immune effector cells & $1 \times 10^{4}$ cell day $^{-1}$ & 1 & 1 \\
\hline$\rho$ & Maximum production rate of effector cells & 1 day $^{-1}$ & 0.3 & 0.8 \\
\hline$\alpha$ & Half saturation constant & $1 \times 10^{6}$ cell & 0.8 & 0.2 \\
\hline$\beta_{1}$ & Effector cells inactivation rate by tumor cells & $1 \times 10^{-8}$ & 1 & 0.2 \\
\hline$\beta_{2}$ & Normal cells inactivation rate by tumor cells & $1 \times 10^{-8}$ & 0.1 & 0.15 \\
\hline$\beta_{3}$ & Tumor cells inactivation rate by effector cells & $1 \times 10^{-8}$ & 0.3 & 0.1 \\
\hline$\beta_{4}$ & Tumor cells inactivation rate by normal cells & $1 \times 10^{-8}$ & 0.3 & 0.1 \\
\hline$d_{1}$ & natural death rate of effector cells & 1 day $^{-1}$ & 0.3 & 0.3 \\
\hline$r_{1}$ & the maximal growth rate of normal cells & 1 day $^{-1}$ & 0.7 & 0.5 \\
\hline$r_{2}$ & the maximal growth rate of tumor cells & 1 day $^{-1}$ & 2.3 & 0.8 \\
\hline$b_{1}$ & Reciprocal of the maximal carrying capacity of normal cells & $1 \times 10^{6}$ cell $^{-1}$ & 0.6 & 0.5 \\
\hline$b_{2}$ & Reciprocal of the maximal carrying capacity of tumor cells & $1 \times 10^{6}$ cell $^{-1}$ & 0.2 & 0.4 \\
\hline$c_{1}$ & the depletion rate of effector cells caused by chemotherapy & $1 \times 10^{6}$ day $^{-1}$ & 0.2 & 0.1 \\
\hline$c_{2}$ & the depletion rate of normal cells caused by chemotherapy & $1 \times 10^{6}$ day $^{-1}$ & 0.2 & 0.1 \\
\hline$c_{3}$ & the depletion rate of tumor cells caused by chemotherapy & $1 \times 10^{6}$ day $^{-1}$ & 0.2 & 0.6 \\
\hline
\end{tabular}

a disease-free steady state $P_{0,1}^{*}$, and $r_{2}-c_{3}-\frac{\beta_{3} s}{c_{1}+d_{1}}-\frac{\beta_{4}\left(r_{1}-c_{2}\right)}{r_{1} b_{1}}=-0.21<0$. Hence, the diseasefree steady state is locally asymptotically stable based on theorem 2 . As illustrated in figure (c), all the trajectories with different initial values converge to the equilibrium point. Time figure (d) shows the quantity of three types cells keep stable after a period of fluctuation. The most exciting phenomenon is that tumor cells disappear because of the effective chemotherapy, which implies that if the efficacy of chemotherapy is good enough, the tumor may still be cured. In fact, there are a small number of similar cases are reported in the clinic. Received a period of chemotherapy, tumor cells cannot be detected in the blood and tissues of some tumor patients. 
(c)

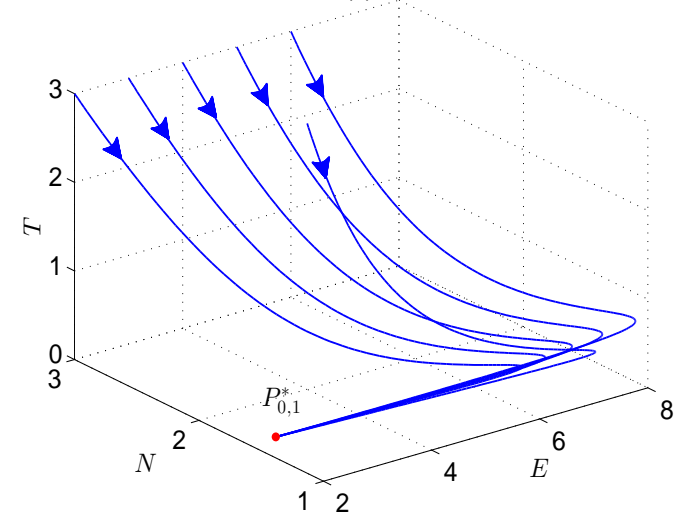

(d)

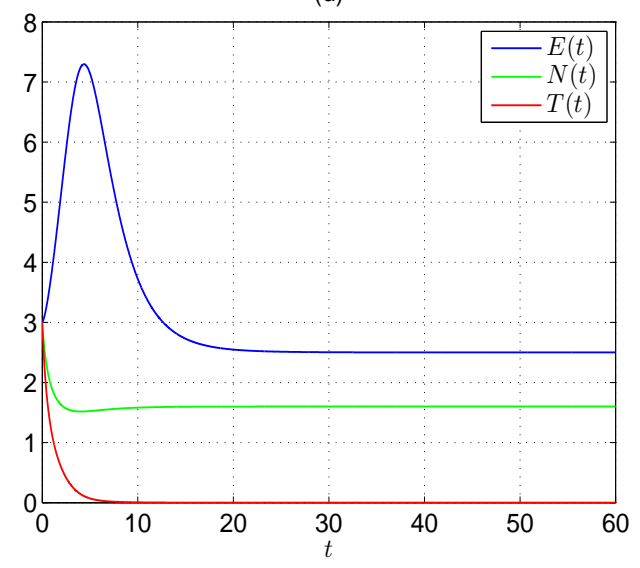

Fig.2. The disease-free equilibrium $P_{0,1}^{*}=(2.50,1.60,0)$ is locally asymptotically stable.In figure (c), the initial values are $(3,3,3),(2,3,3),(4,3,3),(5,3,3),(6,3,3),(4,2,3)$, respectively. The initial value is $(3,3,3)$ in figure $(\mathrm{d})$.

In order to more clearly demonstrate the role of chemotherapy in cancer treatment, we give the bifurcation diagram for the number of tumor cells in response to the change of depletion rate $c_{3}$ as shown in Fig.3. In addition to $c_{3}$, the other parameter values are the same as those in the column of 'Data 1'. As can be observed in Fig.3, the number of tumor cells first drops sharply, and then slowly declines with increasing of $c_{3}$. When $c_{3}$ is approximately equal to 1.6, the quantity of tumor cells first reaches zero. Still, if $c_{3}$ continues to increase, the number of tumor cells keeps in the level of zero. This suggests that the depletion rate of tumor cells caused by chemotherapy could become a decisive factor for the presence or disappearance of the tumor.

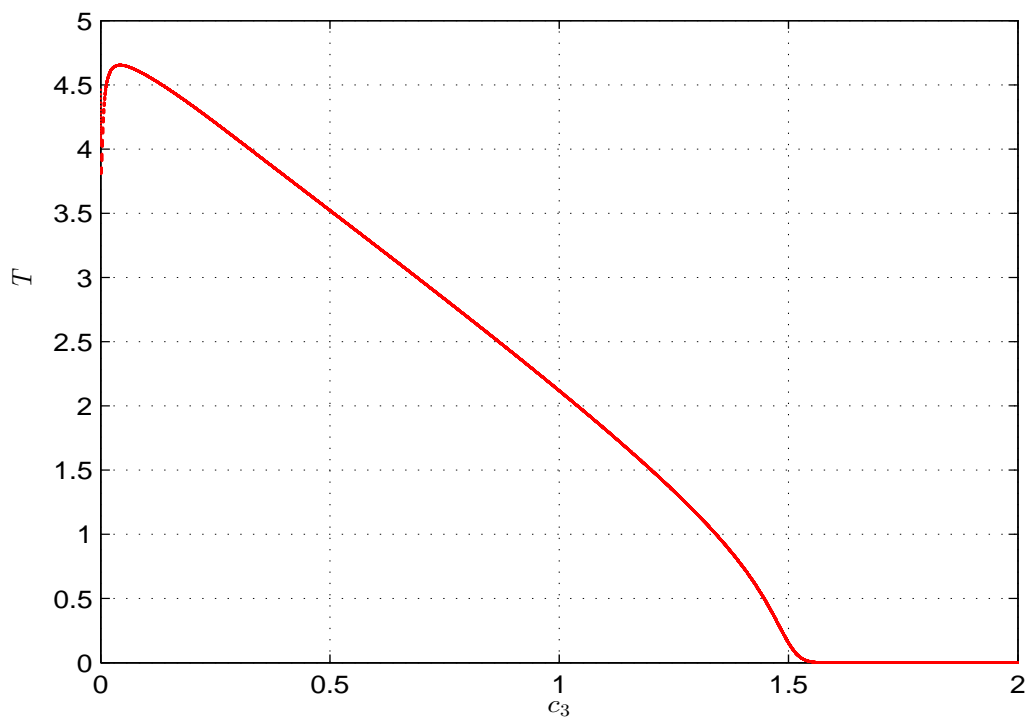

Fig.3. Bifurcation diagram for the number of tumor cells in response to the change of depletion rate caused by chemotherapy. The initial value is $(3,3,6)$ in this figure.

In the following, we further show the impact of stochastic properties on the dynamic behaviors of tumor immune responses. Likewise, taking parameter values from 'Data 1', we make a plot (e) based 
on the stochastic model (14). In fact, the deterministic model shown in figure (b) could be treated as the mean process of the three variables including random errors in figure (e). However, figure (e) represents only a random case for illustration purpose. Without loss of generality, we make a larger number of stochastic samples containing 1000 simulation results. By calculating the mean of these samples, we give the average change trend for the number of three types of cells as shown in figure (f). Obviously, the mean result of a large sample is similar to the situation described by the deterministic model in figure (b).

(e)

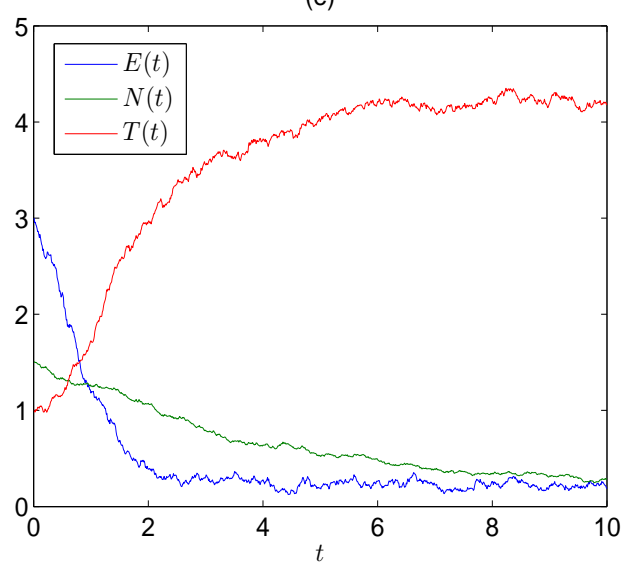

(f)

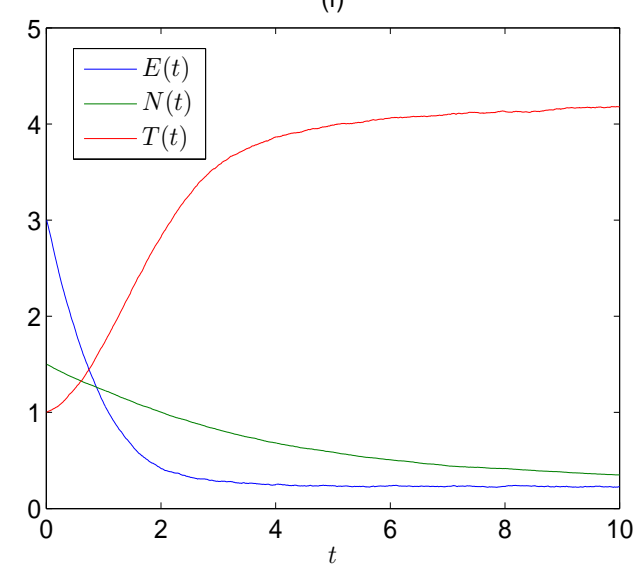

Fig.4. Diagrams for the stochastic (mean) volatility model of $(E(t), N(t), T(t))$. The initial values are both $(3,1.5,1)$ in the two figures.

Besides, residuals of $(E(t), N(t), T(t))$ from the sample above is displayed in figure ( $\mathrm{g}$ ) to check the behaviour of the variance of the three components. Also, the standard deviation of 1000 simulation samples corresponding to each time point of $E(t), N(t), T(t)$ is presented in figure (h). As shown in figure $(\mathrm{g})$, with the achieving of the steady state, the volatility of the three components significantly decrease at different levels. Meanwhile, it is easy to see that the volatility of the three components are not independent, and they more or less have the similar pattern. Surely this phenomenon conforms with the definition of matrix $G$ above.

(g)

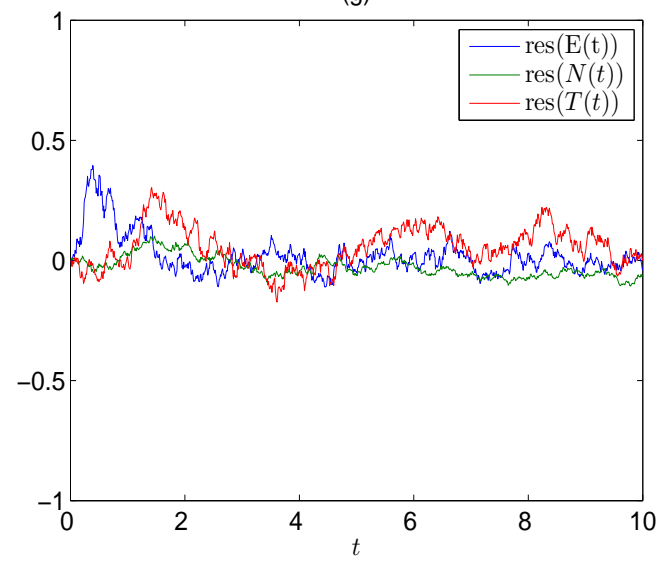

(h)

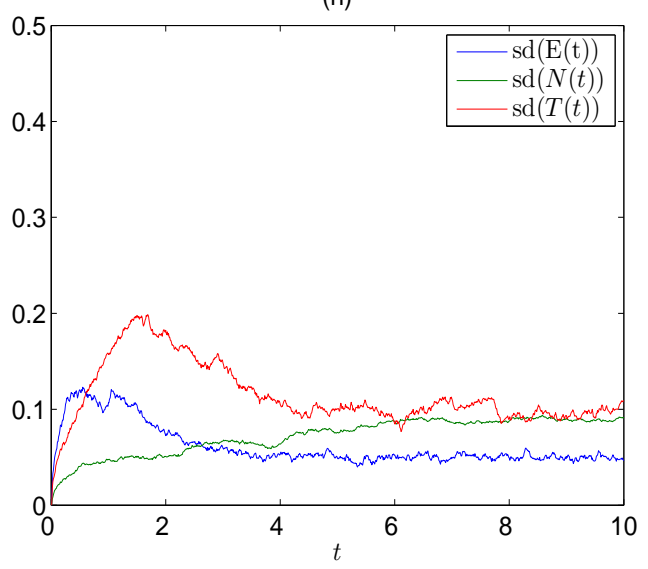

Fig.5. Residuals and standard deviations of $(E(t), N(t), T(t))$ based on the SDE model with Data 1 setting. 
Next, we shall calculate the extinction probability of tumor cells under the setting of 'Data 1'. Given $E(t)=\bar{E}, N(t)=\bar{N}$ and $T(0)=1$, we have the minimum $\tilde{p} \approx 0.8007$ by using equation (30). With different initial tumor cell number $T(0)=m$, we obtain the extinction probability as follows

$$
\lim _{t \rightarrow \infty} \operatorname{Pr}\{\mathrm{T}(\mathrm{t})=0 \mid \mathrm{T}(0)=\mathrm{m}\}=0.8007^{\mathrm{m}} .
$$

In order to demonstrate the relationship between the extinction probability and some important parameters, we make a probability plot in Fig. 6 based on the setting: $r_{2} \in[3,8], c_{3} \in[0.1,0.55]$ and the rest parameter values are the same as themselves in the column of 'Data 1'. Under these parameter values, it is easy to obtain the extinction probability changing between [0.1298, 0.9604]. From Fig.6, it is obvious that the extinction probability of tumor cells will increase with the increase of $c_{3}$, while it will decrease with the increase of $r_{2}$. This also proves that the effect of chemotherapy may decide the growth trend of tumor cells.

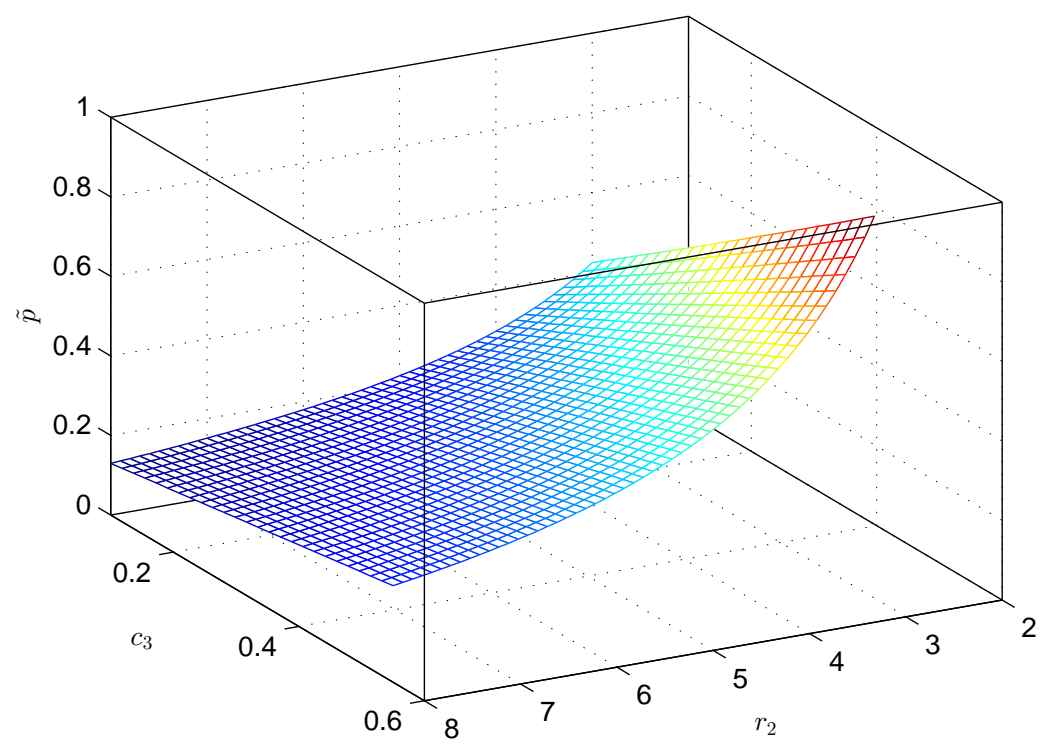

Fig.6. Probability of tumor extinction $\tilde{p}$, giving $E(t)=\bar{E}, N(t)=\bar{N}, T(0)=1, r_{2} \in[3,8]$ and $c_{3} \in[0.1,0.55]$.

\section{Conclusions}

Chemotherapy, as an effective cancer treatment method, lengthens the lives of many cancer patients. The goal of this paper is to explore the dynamics behaviors of tumor immune-responses under chemotherapy. Based on the work of Pillis and Radunskaya [32,33], we first develop an ODE model to describe the quantities' change for tumor cells, normal cells and effector cells by adding the effect of chemotherapy. All solutions of the deterministic ODE model are proven to be positive and ultimately bounded under the initial conditions. The existences of disease-free and positive equilibriums are analyzed by discussing the roots' distributions of a cubic equation. We further show that if the disease-free equilibrium point exists and $r_{2}-c_{3}-\frac{\beta_{3} s}{c_{1}+d_{1}}-\frac{\beta_{4}\left(r_{1}-c_{2}\right)}{b_{1} r_{1}}<0$, then it is locally asymptotically stable, 
while the stability of positive equilibrium point is locally asymptotically stable as long as it satisfies the conditions of existence (see Fig.1 and Fig.2). Actually, the stability of positive equilibrium point explains why cancer patients can maintain a stable station for months to years. Efficient chemotherapy could eliminate cancer cells (see Fig.3), which implies the effect of chemotherapy maybe a key to cure cancer.

Considering the stochastic factors in the process of tumor immune responses, such as the variability in cellular reproduction, growth and death, interspecific competitions, and immune response to chemotherapy, we extend the deterministic model to two stochastic models: one is a stochastic differential equations (SDEs) model, the other is a continuous-time Markov chain (CTMC) model. After obtaining the SDEs model, we respectively compute the expectations and variances both of effector immune cells and normal cells in the absence of disease and disease conditions, and find that they could slightly fluctuate around the expectations which are equal to their equilibriums (see Fig.4 and Fig.5). Based on the theory of branching processes, the CTMC model is constructed and then used to estimate the extinction probability of tumor cells(see Fig.6), which reveals the possible quantitative relationship between the disappear of tumor cell and the important parameter values in the model. Overall, the deterministic and stochastic models are helpful to understand the dynamic behaviors of tumor immune responses, and provide valuable insights for making a treatment programme reasonably.

\section{Acknowledgements}

The authors are very grateful to Dr. Xue Peng for her constructive advice and comments. The authors also thank the anonymous referees and Editor (Prof. Eugene Stanley) for their careful reading, valuable comments and helpful suggestions. This work was partially supported by the National Natural Science Fund of China (No.71420107023, No.71771024, No.11701557, No.71601019), the Training Fund for Talents of Beijing (2015000020124G044), the Fund of China Scholarship Council (201506465053), and Fundamental Research Funds for the Central Universities (FRF-BR-17-008A).

\section{References}

[1] Ahmedin Jemal, Freddie Bray, Melissa M. Center, Jacques Ferlay, Elizabeth Ward, and David Forman. Global cancer statistics. CA: A Cancer Journal for Clinicians, 61(2):69-90, 2011.

[2] Lindsey A. Torre, Freddie Bray, Rebecca L. Siegel, Jacques Ferlay, Joannie Lortet-Tieulent, and Ahmedin Jemal. Global cancer statistics, 2012. CA: A Cancer Journal for Clinicians, 65(2):87-108, 2015.

[3] Marek Bodnar and Monika Joanna Piotrowska. Stability analysis of the family of tumour angiogenesis models with distributed time delays. Communications in Nonlinear Science and Numerical Simulation, 31(1-3):124-142, 2016.

[4] Subhas Khajanchi. Bifurcation analysis of a delayed mathematical model for tumor growth. Chaos, Solitons \& Fractals, 77:264-276, 2015. 
[5] Yueping Dong, Gang Huang, Rinko Miyazaki, and Yasuhiro Takeuchi. Dynamics in a tumor immune system with time delays. Applied Mathematics and Computation, 252:99-113, 2015.

[6] Yueping Dong, Rinko Miyazaki, and Yasuhiro Takeuchi. Mathematical modeling on helper t cells in a tumor immune system. Discrete \& Continuous Dynamical Systems - Series B, 19:55-72, 2014.

[7] F. A. Rihan, D. H. Abdelrahman, F. Al-Maskari, F. Ibrahim, and M. A. Abdeen. Delay differential model for tumour-immune response with chemoimmunotherapy and optimal control. Computational and Mathematical Methods in Medicine, 2014:1-15, 2014.

[8] Fuat Gurcan, Senol Kartal, Ilhan Ozturk, and Fatma Bozkurt. Stability and bifurcation analysis of a mathematical model for tumor $\Vdash$ immune interaction with piecewise constant arguments of delay. Chaos, Solitons \& Fractals, 68:169-179, 2014.

[9] Subhas Khajanchi and Sandip Banerjee. Stability and bifurcation analysis of delay induced tumor immune interaction model. Applied Mathematics and Computation, 248:652-671, 2014.

[10] M. Saleem and Tanuja Agrawal. Chaos in a tumor growth model with delayed responses of the immune system. Journal of Applied Mathematics, 2012:1-16, 2012.

[11] Shaoli Wang, Shuli Wang, and Xinyu Song. Hopf bifurcation analysis in a delayed oncolytic virus dynamics with continuous control. Nonlinear Dynamics, 67(1):629-640, 2012.

[12] Alberto D’ Onofrio, Urszula Ledzewicz, Helmut Maurer, and Heinz Schattler. On optimal delivery of combination therapy for tumors. Mathematical Biosciences, 222(1):13-26, 2009.

[13] Radouane Yafia. Hopf bifurcation in differential equations with delay for tumor-immune system competition model. SIAM Journal on Applied Mathematics, 67(6):1693-1703, 2007.

[14] Liuyong Pang, Zhong Zhao, and Xinyu Song. Cost-effectiveness analysis of optimal strategy for tumor treatment. Chaos, Solitons \& Fractals, 87:293-301, 2016.

[15] Liuyong Pang, Lin Shen, and Zhong Zhao. Mathematical modelling and analysis of the tumor treatment regimens with pulsed immunotherapy and chemotherapy. Computational and Mathematical Methods in Medicine, 2016, 2016.

[16] F. A. Rihan, D. H. Abdel Rahman, S. Lakshmanan, and A. S. Alkhajeh. A time delay model of tumourimmune system interactions: Global dynamics, parameter estimation, sensitivity analysis. Applied Mathematics and Computation, 232:606-623, 2014.

[17] L. G. De Pillis, W. Gu, K. R. Fister, T. Head, K. Maples, A. Murugan, T. Neal, and K. Yoshida. Chemotherapy for tumors: An analysis of the dynamics and a study of quadratic and linear optimal controls. Mathematical Biosciences, 209(1):292-315, 2007.

[18] Mehmet Itik, Metin U. Salamci, and Stephen P. Banks. Optimal control of drug therapy in cancer treatment. Nonlinear Analysis: Theory, Methods \& Applications, 71(12):e1473-e1486, 2009.

[19] Ram Rup Sarkar and Sandip Banerjee. Cancer self remission and tumor stability-a stochastic approach. Mathematical Biosciences, 196(1):65-81, 2005. 
[20] G. Albano and V. Giorno. A stochastic model in tumor growth. Journal of Theoretical Biology, 242(2):329336, 2006.

[21] Thomas Bose and Steffen Trimper. Stochastic model for tumor growth with immunization. Physical Review E, 79(5):051903, 2009.

[22] Yong Xu, Jing Feng, JuanJuan Li, and Huiqing Zhang. Stochastic bifurcation for a tumor-immune system with symmetric lévy noise. Physica A: Statistical Mechanics and its Applications, 392(20):4739-4748, 2013.

[23] Kwang Su Kim, Sangil Kim, and Il Hyo Jung. Dynamics of tumor virotherapy: A deterministic and stochastic model approach. Stochastic Analysis and Applications, 34(3):483-495, 2016.

[24] Joan H. Schiller, David Harrington, Chandra P. Belani, Corey Langer, Alan Sandler, James Krook, Junming Zhu, and David H. Johnson. Comparison of four chemotherapy regimens for advanced non-small-cell lung cancer. New England Journal of Medicine, 346(2):92-98, 2002.

[25] Pietro Marino, Sandro Pampallona, Armando Preatoni, Alessandra Cantoni, and Fulvio Invernizzi. Chemotherapy vs supportive care in advanced non-small-cell lung cancer: results of a meta-analysis of the literature. CHEST Journal, 106(3):861-865, 1994.

[26] W. Alberti, G. Anderson, A. Bartolucci, and D. Bell. Chemotherapy in non-small cell lung cancer: a metaanalysis using updated data on individual patients from 52 randomised clinical trials. British Medical Journal, 311(7010):899-909, 1995.

[27] Roberto Grilli, Andrew D. Oxman, and Jim A. Julian. Chemotherapy for advanced non-small-cell lung cancer: how much benefit is enough? Journal of Clinical Oncology, 11(10):1866-1872, 1993.

[28] M. H. Cullen, L. J. Billingham, C. M. Woodroffe, A. D. Chetiyawardana, N. H. Gower, R. Joshi, D. R. Ferry, R. M. Rudd, S. G. Spiro, and J. E. Cook. Mitomycin, ifosfamide, and cisplatin in unresectable non-small-cell lung cancer: Effects on survival and quality of life. Journal of Clinical Oncology, 17(10):3188-3194, 1999.

[29] R. P. Abratt, W. R. Bezwoda, L. Goedhals, and D. J. Hacking. 1086 a phase ii study of gemcitabine with cisplatin in patients with non-small cell lung cancer. European Journal of Cancer, 31:S226, 1995.

[30] L. Crino, G. Scagliotti, M. Marangolo, F. Figoli, M. Clerici, F. De Marinis, F. Salvati, G. Cruciani, L. Dogliotti, and F. Pucci. Cisplatin-gemcitabine combination in advanced non-small-cell lung cancer: a phase ii study. Journal of Clinical Oncology, 15(1):297-303, 1997.

[31] Corey J. Langer, John C. Leighton, Robert L. Comis, Peter J. O’Dwyer, Cecilia A. McAleer, Colleen A. Bonjo, Paul F. Engstrom, Samuel Litwin, and Robert F. Ozols. Paclitaxel and carboplatin in combination in the treatment of advanced non-small-cell lung cancer: a phase ii toxicity, response, and survival analysis. Journal of Clinical Oncology, 13(8):1860-1870, 1995.

[32] Lisette G. De Pillis and A. Radunskaya. The dynamics of an optimally controlled tumor model: A case study. Mathematical and Computer Modelling, 37(11):1221-1244, 2003.

[33] L. G. DePillis, A. Eladdadi, and A. E. Radunskaya. Modeling cancer-immune responses to therapy. Journal of Pharmacokinetics and Pharmacodynamics, 41(5):461-478, 2014. 
[34] V. A. Kuznetsov. A mathematical model for the interaction between cytotoxic t lymphocytes and tumour cells. analysis of the growth, stabilization, and regression of a b-cell lymphoma in mice chimeric with respect to the major histocompatibility complex. Biomedical Science, 2(5):465-476, 1990.

[35] Vladimir A. Kuznetsov, Iliya A. Makalkin, Mark A. Taylor, and Alan S. Perelson. Nonlinear dynamics of immunogenic tumors: parameter estimation and global bifurcation analysis. Bulletin of Mathematical Biology, 56(2):295-321, 1994.

[36] Theodore E Harris. The theory of branching processes. Courier Corporation, 2002.

[37] Edward J Allen, Linda JS Allen, Armando Arciniega, and Priscilla E Greenwood. Construction of equivalent stochastic differential equation models. Stochastic Analysis and Applications, 26(2):274-297, 2008.

[38] Kiyosi Itô. Stochastic integral. Proceedings of the Imperial Academy, 20(8):519-524, 1944.

[39] Bernt Oksendal. Stochastic differential equations: an introduction with applications. Springer Science \& Business Media, 2013.

[40] Andrei Kolmogoroff. Über die analytischen methoden in der wahrscheinlichkeitsrechnung. Mathematische Annalen, 104(1):415-458, 1931.

[41] Samuel Karlin. A first course in stochastic processes. Academic press, 2014.

[42] Theodore E. Harris. The theory of branching processes. Courier Corporation, 2002.

[43] Christophe Letellier, Fabrice Denis, and Luis A. Aguirre. What can be learned from a chaotic cancer model? Journal of Theoretical Biology, 322:7-16, 2013.

[44] Motahareh Moghtadaei, Mohammad Reza Hashemi Golpayegani, and Reza Malekzadeh. Periodic and chaotic dynamics in a map-based model of tumor-immune interaction. Journal of Theoretical Biology, 334:130$140,2013$.

[45] Lisette G. de Pillis, Weiqing Gu, and Ami E. Radunskaya. Mixed immunotherapy and chemotherapy of tumors: modeling, applications and biological interpretations. Journal of Theoretical Biology, 238(4):841$862,2006$. 